\title{
Interview with John Dunlop: Shape-Changing Materials
}

KK: You are working on the activity of 'living' as well as 'dead' materials, especially with phenomena of shape changing and the possibility of a bioinspired transfer from shapechanging mechanisms in living systems to synthetic systems. Many of your studies belong to the field of active matter research, although you do not explicitly refer to this concept in your publications. May I ask what your understanding is of active matter? And how as a scientist did you find your way into this relatively new field of research?

JD: I suppose I should start with the way I got into active matter. It started from the perspective of my $\mathrm{PhD}$, where I was interested in processes of changing morphology of the internal structure of metals. ${ }^{1}$ In the project, we were trying to understand how crystals grew and changed shape due to different energy inputs. For example, if you deform a material, you can store lots of energy from the deformation process, and this is then transformed, if you supply enough heat to the surroundings, into a process by which crystals will change size, orientation, shape, and so on. We were trying to understand these processes and develop simple physically based models to describe their behavior. The idea was that if we could determine a relationship between structure and mechanical function, and if we could understand how structure changes with temperature, for example, we could then predict how mechanical properties of the material would evolve. Subsequently, in February 2006, I started working in the Department of Biomaterials at the Max Planck Institute of Colloids and Interfaces (MPICI) in Potsdam. ${ }^{2}$ It took a little bit of time but I began to become interested in processes of describing structural shape changes initially in bones, but shortly afterward also in plants. These two processes of shape change have a commonality with what I was looking at before in that we were trying to come up with very simple descriptions of how in the architecture of trabecular bone, for example, shape and orientation changes in response to the external environment [Dunlop and Fratzl 2013;

1 John Dunlop did his PhD on internal variable modeling of creep and recrystallization in zirconium alloys in the years 2002-2005 at the Laboratoire de Thermodynamique et de Physico-chimie des Matériaux of the Institut national polytechnique de Grenoble in France [Dunlop, Bréchet, and Legras 2004; Zurob, Bréchet, and Dunlop 2006].

2 In 2006, Dunlop continued his research at the MPICI in Potsdam (Germany), first as a postdoctoral scientist and in 2007 also as Alexander von Humboldt Research Fellow. From 2008 to 2017, he led his own research group ‘Biomimetic Actuation and Tissue Growth’ at the MPICI [Dunlop 2021].

Acknowledgment: Karin Krauthausen acknowledges the support of the Cluster of Excellence 'Matters of Activity. Image Space Material' funded by the Deutsche Forschungsgemeinschaft (DFG, German Research Foundation) under Germany's Excellence Strategy - EXC 2025 - 390648296. 
Gamsjäger et al. 2013; Fratzl and Weinkamer 2007]. In terms of plant tissues, this was a work done together with the research group of Ingo Burgert at the time. ${ }^{3}$ That was an attempt to describe how objects such as the pine cone or the ice plant change shape in accordance with changes in the environment, changes in humidity or water content, for example (see Fig. 1) [Harrington et al. 2011; Burgert and Dunlop 2011].

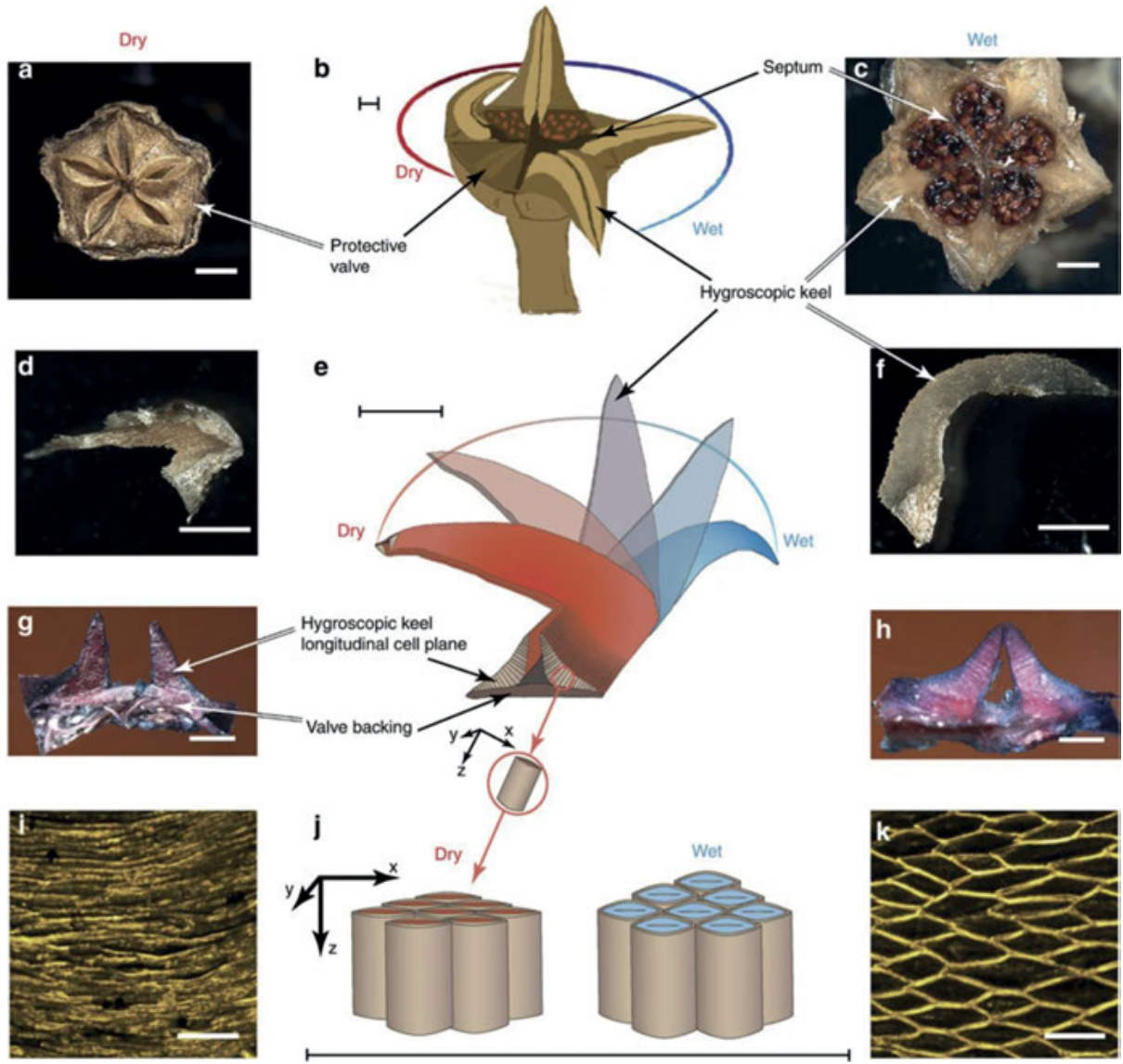

Fig. 1: Hygroscopic opening of the ice plant seed capsule is controlled by tissue microstructure. Illustration of the different levels of architectural complexity in the ice plant seed capsule at different length scales $(a-k)$. The images on the left-hand side show the different tissue structures in the dry state and on the right-hand side in the wet state. The central column gives schematic illustrations of the different tissues. Scale bars are defined as: $a$ and $c=2 \mathrm{~mm} ; b, e$, and $j \approx 1 \mathrm{~mm} ; d$ and $f=1 \mathrm{~mm} ; g$ and $h=0.5 \mathrm{~mm} ; i$ and $k=0.1 \mathrm{~mm}$. Reprinted by permission from Springer Nature: [Harrington et al. 2011, fig. 1].

3 Until 2011 Ingo Burgert led the research group 'Plant Biomechanics and Biomimetics' at the MPICI in Potsdam (Germany) (https://www.mpikg.mpg.de/5788791/plant-biomechanics-andbiomimetics, accessed May 5, 2021). 
Through that I got into this field of active matter. This continued during my time at the MPICI and is also being carried out here in my research group 'MorphoPhysics' at the Paris Lodron University of Salzburg. ${ }^{4}$ Returning to the question of what I consider active matter to be in this context, I judge it as being a class of materials that respond to internal energy (coming from cells acting in response to their environment), or to energy that in principle comes from the external environment. These materials use that energy to change their structure, their arrangement. This could be an internal or an external structure. I see it as being a fairly broad topic that would encompass the kinds of shape changes you get in objects that can swell and change form in their environment. I could also see it as being in processes where cells act locally in response to their environment. You get some sort of collective behavior. You can also observe this sort of collective behavior in general. This is what you have in the descriptions of swarm behavior, the flocking of birds or insects [Vicsek et al. 1995; Grégoire and Chaté 2004]. All of these would also fit in this perspective. My view in this field is really about thinking of materials as such, and how they change their structural shape in response to their environment.

KK: If you look back at the development of your research on shape-change phenomena (and in this sense on active matter), which influences, which disciplines, and which tools were important for you and helped you on the way?

JD: My background is varied in that I studied materials engineering and chemistry. The materials engineering or materials science background is one where you take concepts from physics or chemistry, and then you apply them to understand the object you are interested in as an engineer. Through the materials science background, you have quite a good overview of the different fields. Chemistry gives you a good molecular understanding of what is going on. But the most important part would be the physics of biological systems. It is there that these concepts of emergent phenomena have become more mainstream over the last 10 to 15 to 20 years. ${ }^{5}$ The descriptions of these phenomena and the tools used have become very helpful for research, particularly in the field of active matter.

From another standpoint, you could say that there are basic tools of engineering, for instance the simulation tools that we use. Having an understanding of mechanics and of basic physical phenomena such as diffusion and similar sorts of things is also pretty important. And having the opportunity to talk to people from other disciplines

\footnotetext{
4 Since 2017, Dunlop is professor of biological physics at the Department of the Chemistry and Physics of Materials at Paris Lodron University of Salzburg, where he leads the research group 'MorphoPhysics' (https://www.morphophysics.com/john-dunlop, accessed May 5, 2021). For the most recent research in the fields of actuation in plants and tissue growth, see: [Dunlop et al. 2020; Ehrig et al. 2019].

5 For an excellent introduction into models of self-organization, see: [Camazine et al. 2003].
} 
is crucial, like talking to biologists in order to understand the important features that you observe in a cell culture or in an organism.

Perhaps the most central point for me would be the training I acquired in materials science where you describe, for example, the phenomena you see and then try and come up with relatively simple models to represent the physical principles that are governing any phenomena that you are observing. Even if the models themselves are not fully successful in describing everything, you still learn a lot - if you keep their limitations in mind. I think the materials sciences are quite strong in this respect, because they really do bring these simplified models from chemistry and physics together in order to describe applied problems. This is relatively easy to transfer to the description of biological systems, at least from a physical viewpoint.

KK: The research field of active matter has developed strongly during the past 20 to 30 years. ${ }^{6}$ It is supported by different disciplines and probably expresses a broader interest among the sciences in the activity of materials. Today, the research on active matter seems to be quite diversified. For a scientist working in this field, is it still possible to get the bigger picture?

JD: Getting an overview of the entire field is probably very difficult or unrealistic, especially if you want it to be detailed. But what you can certainly do is to get a relatively broad overview of key types of phenomena that I would say fit into the field of active material or matter research. By calling it 'materials' here, my own background is coloring my discourse about it. You can divide the field into subtopics or sub-foci. You can go in the direction of emergent phenomena or patterning - that is already a large field in itself [Camazine et al. 2003]. But it has been shown in quite a few biological systems that you can describe patterning of pigmentation in the skin or patterning of coloring of shells and things like that by relatively simple reaction-diffusion equations [Nüsslein-Volhard and Singh 2017; Kondo 2002; Turing 1952; Malacincski and Bryant 1984; Meinhardt and Klingler 1987]. To give you an idea, we can consider a chemical system in which we have a pigment whose production is stimulated by the presence of component A (this species is autocatalytic, meaning it stimulates its own production) but is inhibited by the presence of component B. Under situations where these components are well mixed, the system will tend to a stable steady state, meaning the system will present either a pigment or not. When the system is not well mixed, the spatial diffusion of species A and B can give rise to spatially inhomogeneous patterns, leading to complex pigmentation patterns in the system. It has been shown that these types of models can also apply to biological systems at the level of the cell up to the organism.

From the perspective of an overview, it is also important to highlight the role of mechanics in active matter, in that much of what we describe as being active matter

6 For a good introduction in the different field of active matter research, see: [Gompper et al. 2020]. 
consists of the interaction of objects and materials through forces [Ambrosi et al. 2011; Trepat and Sahai 2018]. As I see it, that is a key point. The description of shape changes, or the description of how one goes about changing the shape of an object or transforming its internal structure, implies that you need to give a detailed account of forces, of how mathematically you can describe a shape. You need to be able to describe how a surface changes its geometry, how its internal pattern changes with time or in response to a signal [Kollmannsberger et al. 2011; Guiducci et al. 2015; Ehrig et al. 2019]. I believe that is another component that is very important. And then perhaps there is the applied direction, which would be examples of these sorts of changes, patterning, and how that fits with the first two.

If you can nail down what you mean by active matter, then you can get an overview. But of course, by going in-depth with each example, you can very quickly get lost in the details. And also, I guess, the other problem is that it is such a broad topic that you can find many examples in the biological, physical, or mathematical literature. It can be very difficult to get into active matter, especially when you come from a different field. Being a newbie in the field is somewhat tough to digest. It is possible, however, to get an overview of the important themes.

KK: Would you say that active matter works as an umbrella term or migrating concept that is able to 'bridge' between different disciplines?

JD: For sure. For example, from the experience of the research I have been conducting on shape changing, you end up using these different tools in widely different disciplines. For example, when we explored macroscopic shape changes of the ice plant or the Banksia seed pods, we used the same computational tools that we use to describe the behavior of synthetic materials [Guiducci et al. 2015; Harrington et al. 2011, footnote 6; Huss et al. 2019]. Furthermore, the description of the reaction-diffusion equations mentioned earlier works well in chemistry, but it can also be used in biology. The physics of this is also very interesting and can also be characterized in this respect [Halatek and Frey 2018]. There is certainly a lot of commonality there. To view it as a bridge makes a lot of sense.

KK: You mentioned the role of energy in active materials. Gautam I. Menon in his 'tentative' (as he called it) definition of active matter also referred to energy from an internal source or as a trigger from the environment for explaining activity. ${ }^{7}$ In Menon's account, active matter is understood as a far-from-equilibrium system that can show emergent behavior. Hence, he refers often to living systems whose behavior is

7 See [Menon 2010, p. 194]: "Active matter is a term which describes a material (either in the continuum or naturally decomposable into discrete units), which is driven out of equilibrium through the transduction of energy derived from an internal energy depot or ambient medium into work performed on the environment. Such systems are generically capable of emergent behaviour at large scales.” 
explained by thermodynamic theories and statistical physics. In your own research on active materials, you often use mathematical descriptions, for instance the geometry of an internal or external structure, in order to explain either the growth of cells or the actuation in plant materials. Could you tell me more about the importance of geometry for the explanation of activity?

JD: In order to answer this question, it is necessary to describe the two directions that interest me. On the one hand, I am into changes in environmental conditions and I suppose in this respect this is inspired by these structures that you can see in plants that actuate, like a pine cone (see Fig. 2), wheat awns, the ice plant, or the Banksia seed pods [Dunlop, Weinkamer, and Fratzl 2011; Harrington et al. 2011; Huss et al. 2019].
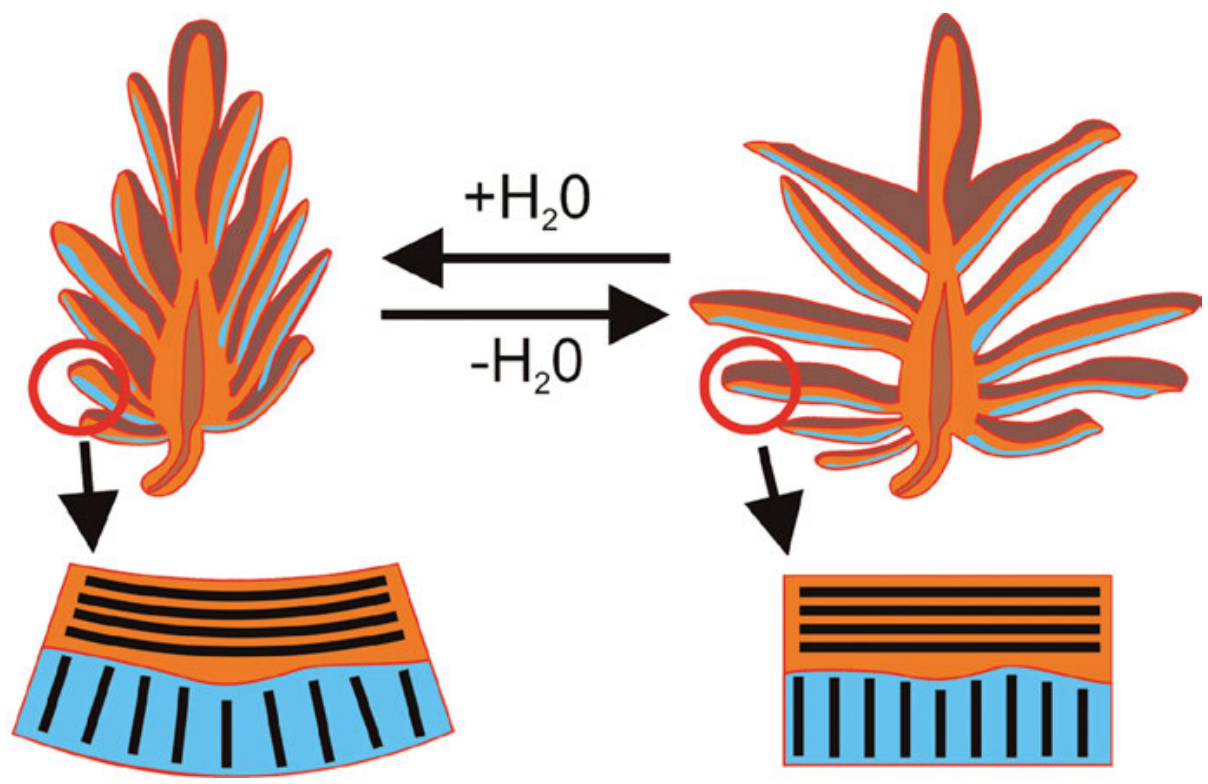

Fig. 2: Hygroscopic opening of pine cones. These cones are closed when wet and open when dry for seed release. Sketches of how different orientations of the cellulose microfibrils inside different layers gives rise to bending motion upon changes in moisture content. From: [Dunlop, Weinkammer, and Fratzl 2011, p. 76, fig. 7]. License: CC BY-NC-ND 3.0 (https://creativecommons.org/licenses/bync-nd/3.0/).

These actuation processes occur in a dead material, so there is no metabolism going on anymore in these objects. The seeds of course are still living, but the material surrounding them is dead. Somehow, the activity or potential for activity is in-built into the structure, and when a stimulus comes along certain components will swell or change their volume. They do it within the constraints of the surrounding tissues that maybe do not swell so much or maybe more. Depending on how these are arranged 
in space, you can get very different types of 3D movements and shape changes. What interests me is to sort of step back a little bit. Hence, on the one hand to work together with experimentalists who study these sorts of plant-based materials or observed structures and to assist them in sort of analyzing the process of movement and actuation that can be observed, but on the other hand to take a step back and ask how the geometric arrangement of say swellable and non-swellable components controls the macroscopic shape change that you observe. And, of course, how you can use that in a design process. For example, if you say you want to have a certain shape change, is there a unique way of achieving it? In principle, there are different ways of achieving macroscopic deformation. What is interesting is that it turns out that there is no unique solution for a particular shape change [Turcaud et al. 2011; Reyssat and Mahadevan 2009; Fratzl and Barth 2009]. Thus, you can have the classic example of a bilayer where you can have a sharp interface between material A and material B, and that will give a certain curvature. But you can also introduce a gradient to your material properties going from material A to material B with no sharp interface, and it can give you the same curvature as well. You simply have to play with the swellability of the different components in the right way. I guess trying to explore these is all one thing. What is very important for understanding this is a description of the mechanical properties of a material, because you need the elastic modulus to describe how stress relates to strain or force relates to displacement in the material. And then you need a description of how the material changes its volume with respect to some external stimulus, whether the latter is temperature or swellability due to changes in humidity or something of the sort. Hence, you have something that depends on the stimulus and you have your mechanical properties, and then you can in principle take tools that you use for engineering - such as the finite element method, a numerical method for solving partial differential equations in two or three space variables [Zienkiewicz et al. 2005] - and then stimulate your objects and see how they change shape and form. The geometry comes in the context of boundary conditions. Not only do you have shape change of some composite materials consisting of different components but you also have the fact that, if you constrain an object inside some physical environment, you will then influence the sorts of shape changes that are possible - and you will also push the shape changes to go in certain directions versus in others. In some of the work that we were doing together with Leonid Ionov from the Leibniz Institute of Polymer Research in Dresden (Germany), we were observing that by spatially controlling the speed at which an environmental signal comes into effect - in this case the diffusion of water through its bilayers - you could control the process by which your actuation occurs and you can push your actuation in certain directions, which would not necessarily be the lowest energy configuration (see Fig. 3) [Stoychev et al. 2012; Stoychev et al. 2013].

In this way, by playing with boundary conditions - which by definition means playing with the geometry of the surroundings - you can then influence your shape changes. This is also a very interesting direction to go. In this sense, you study materials which 

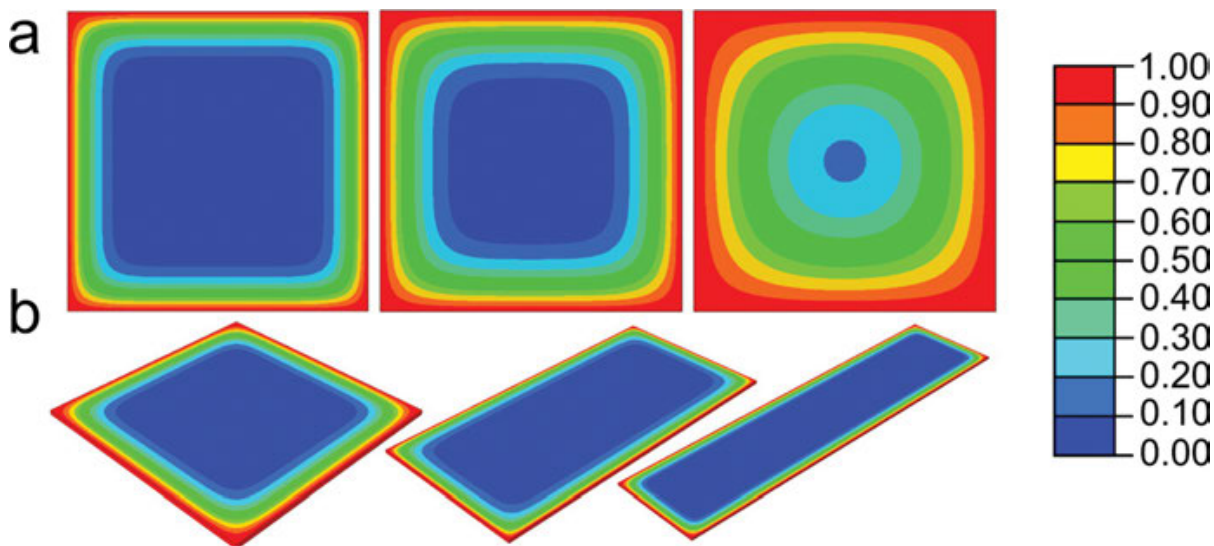

C

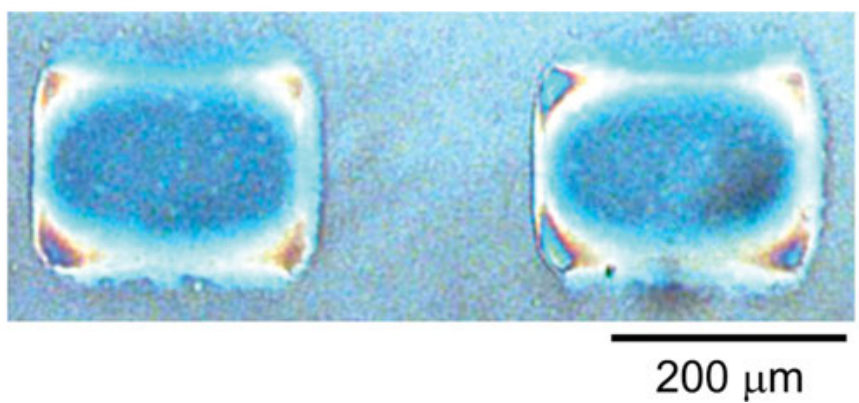

Fig. 3: Color map of the calculated swelling (from 0 to 1) controlled by water diffusion in the active monolayer with a lateral constant boundary condition (blue is non-swollen) dependent on (a) time and (b) shape obtained by finite element simulations as well as (c) experimentally obtained microscopy snapshot of swollen P(NIPAM-BA) - PMMA bilayer after a few seconds of swelling. From: [Stoychev et al. 2012, p. 3930, fig. 6]. (C) 2012, American Chemical Society.

have the potential for activity built in, and it is the arrangement of the materials that determine in what direction shape changes will occur.

KK: That is one focus of your research. And the other focus?

JD: The other focus goes in the direction of living systems. The goal is to understand how the shape of the environment controls processes of growth, processes of cell migration, processes of cell patterning. When I started off as a postdoc in Peter Fratzl's Department of Biomaterials at the MPICI in Potsdam (Germany), I met Monika Rumpler from the Ludwig Boltzmann Institute of Osteology in Vienna (Austria), who was using 3D printing to create scaffolds for tissue engineering. From a certain perspective, this was a very applied topic as the ultimate goal would be to build a scaffold that you can implant in a fracture gap in bone and then hopefully create an ideal environment to stimulate cells to move in and produce new bone. What Monika did was that she first started looking at rather complex scaffolds with multiple interconnections, with high 
porosity. The research group could show that the cells would grow inside these scaffolds, but it was difficult to really observe individual holes or pores inside these scaffolds and what was going on there. They then had the great idea to use rapid prototyping or 3D printing to create single pores or single holes where you can explore the role of shape and the shape of this pore on how cells grow and pattern [Rumpler et al. 2008]. What got me excited when I was working with them was that the structures that appeared in these pores seemed describable with very simple models - either models which you normally use to describe, for instance, the shape of fluids on surfaces, or models that you use to describe crystal growth and similar things coming from the materials sciences or physics (see Fig. 4) [Dunlop et al. 2010; Bidan et al. 2012; Gamsjäger et al. 2013; Fischer et al. 2015; Lecuit and Lenne 2007]. I guess it was one of these strange eureka-style moments where you sit down and start doing some calculations and you realize we get simulated shapes that match very well the shapes of the experiments. This is how I got into this topic. I began to become interested firstly in developing theoretical models to understand how cells interact with surfaces and shapes where the shapes are much bigger than the size of individual cells. And then with time I got more and more into designing experiments to test models, and then there was this nice feedback process between experimenting, simulating, and modeling.

When one thinks about the role of geometry, I guess the topic or our interest was how do the external boundary conditions of a growing tissue, hence a growing set of cells - how does this shape influence the collective behavior of cells, inside or on these objects? Can you come up with models that give you an average behavior of cells and tissues at a variety of different length scales that you can then travel back and link to experiments? There is a lot to be done related to understanding how these geometric boundary conditions influence cells. So, what is the signaling process? Is it that cells are attached to the substrate and can then somehow sense local geometric features like local curvature? When we talk about length scales that are much larger than the single cell, the cells in principle would not respond to this. This is something that you can only get when many cells operate together, and then you arrive at this whole field of collective behavior. I think that is where these models of swarming behaviors could be very interesting.

KK: This mathematical modeling of growth processes reminds me of D'Arcy Wentworth Thompson's On Growth and Form (1917/1942), where he wanted to unify biology, physics, and mathematics in order to find universal laws for the morphology and structural development of living beings. Of course, with today's mathematical tools and high-tech instruments like scanning electron microscopes, this is a different situation. Could your research on 'MorphoPhysics' (as well as the research being carried out by other scientists in this field) possibly lead to a geometry of growth processes in living materials?

JD: In some sense, I suppose. There are several questions that would be behind it. What we saw, at least in our first experiments, is that you could very well describe 

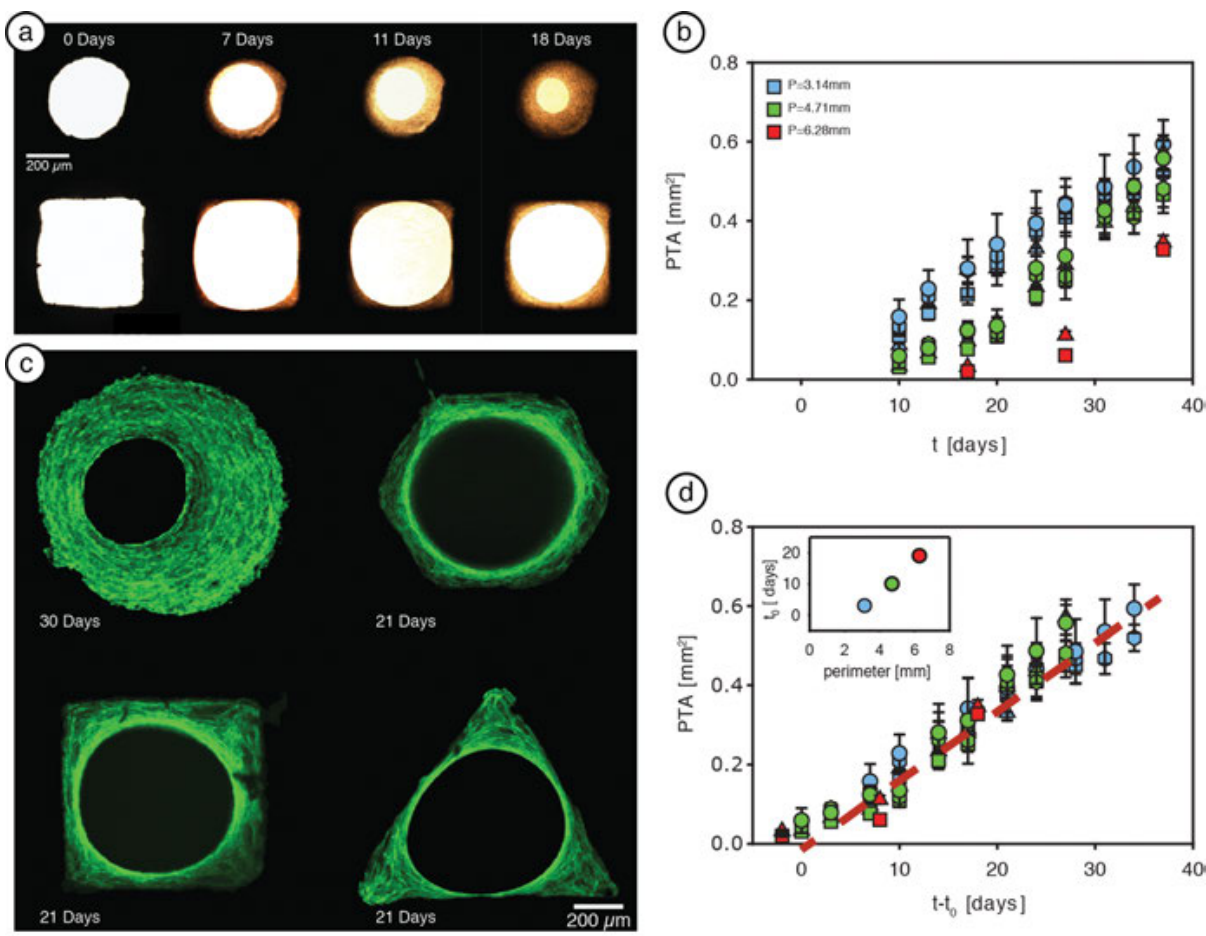

(d)
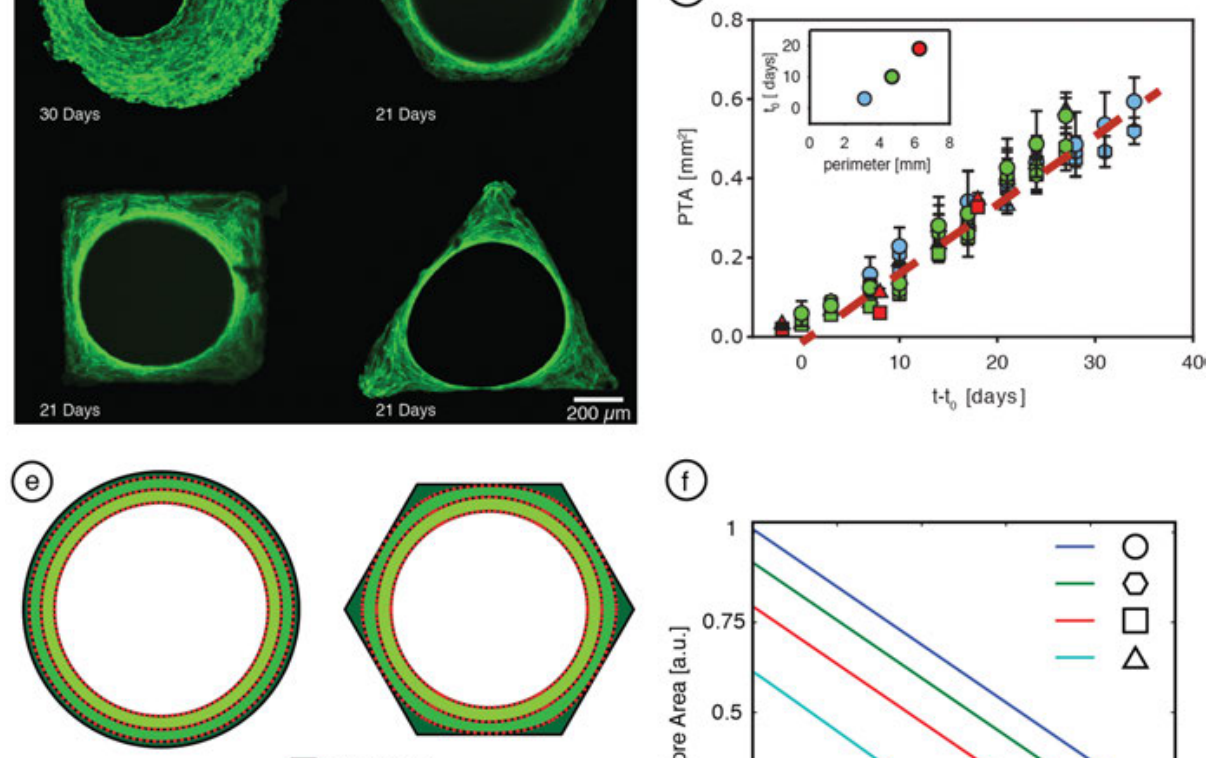

(f)
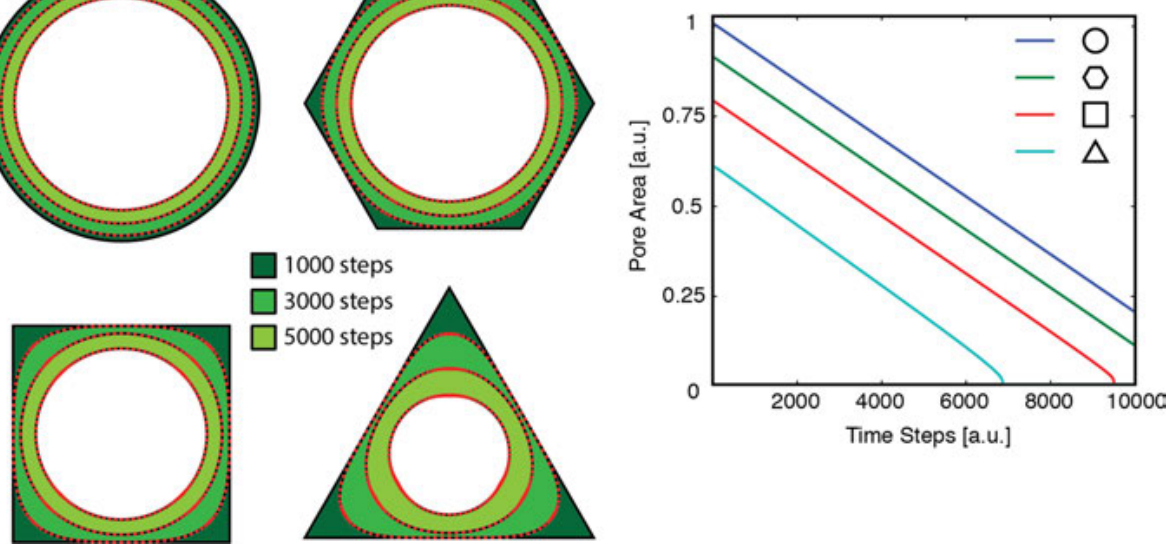

Fig. 4: Experiments and simulations of tissue growth in convex pores. (a) Phase contrast microscopy images of two pores within hydroxyapatite (HA) scaffolds during MC3T3-E1 cell culture experiments. Tissue appears brown, scaffold is black, and medium appears white (images produced by C. Bidan). (b) Projected tissue area as a function of culture time for four different pore cross sections (circular, hexagonal, square, and triangular) and three different pore perimeters (3.14, 4.71, and $6.28 \mathrm{~mm}$ ). Error bars indicate standard error $(n=10)$ (data from [Rumpler et al. 2008]). (c) Laser scanning confocal microscopy images of four different HA pores containing tissue 
the shape changes with models that are essentially geometric descriptions. Thus, this curvature-driven growth model that we had is a model that takes an interface, which can have a varying curvature, and you would allow that shape to change or move as a function of that local curvature [Rumpler et al. 2008; Bidan et al. 2012; Dunlop et al. 2010]. The simplest version of such a model is that a point on an interface moves at a rate directly proportional to its local curvature. You then calculate the motion for all points on the interfaces and then move them. This then gives you a new shape, which would subsequently change iteratively. This works quite well up to a certain point, at least in the system we were working with or the systems we have looked at. The question then is: what is actually controlling it or why is it that in the majority of our experiments after about 20 or 25 days of cell culture the growth process starts slowing down? What is going on? Is this a process by which the cells are differentiating, gaining new characteristics so that they are not behaving in the same way they were earlier on in the culture, or is it a process by which the geometry has changed so much that there is this feedback effect, the cells have created a geometry where they do not want to grow anymore? And then there is also the question of what is responsible for this geometry sensing. How are the cells actually doing it? Are they doing it by means of collective behavior, because they are attached to each other, glued to each other by these adhesive molecules? Why do the cells then start exerting tension on their surroundings? Is this due to their cytoskeleton? They start pulling on each other. The underlying causes are to be understood in the direction of mechanics or forces between interacting objects in a constrained environment.

KK: In the description of growth processes or actuation phenomena, there are different explanations involved that come from different disciplines like physics, biology, and chemistry, as well as mathematics. I wonder how these different understandings can come together. Joanna Aizenberg and Alison Grinthal from Harvard University, in a paper on responsive materials from 2013, wrote that the microarchitecture of living materials should not be conceived as a static configuration or a passive scaffold but more like a "complex multi-scale feedback dance" [Grinthal and Aizenberg 2013, p. 7073]. In their article, they also call it a "dense interconnected jungle” of

\footnotetext{
Fig. 4 (continued)

produced by MC3T3-E1 cells in which actin has been stained (green) with phalloidin-FITC. The image of the circular pore contains tissue grown for 30 days; the other pores show images after 21 days of culture. (d) The data from (b) is replotted as a function of culture time minus lag time, which is the time taken for linear growth to start. The red dotted line indicates the linear growth of tissues on hydroxyapatite scaffolds in convex-shaped pores. (e) Predicted tissue geometries according to the curvature-driven growth model [Rumpler et al. 2008] at three different time points. Each shape has the same starting perimeter. (f) Calculated remaining pore area (white) as a function of time for the simulations shown in (e) From: [Dunlop 2015, p. 20, figs. 2-6]. (C) John Dunlop.
} 
"reactants and catalysts" - to which one could maybe add: of forces and environmental conditions - hence very different signaling. ${ }^{8}$

JD: I like the imagery of that. There are quite a few groups that are working in this direction, especially in the context of regenerative medicine or in cell cultures - one could mention Anja Geitmann, Christopher Chen, Celeste Nelson, Xavier Trepat, Dennis Discher, Carl-Philipp Heisenberg, Viola Vogel, to name just a few. These groups are trying to understand what is the role of mechanics and what is the role of chemistry or biochemistry on cell behavior or tissue behavior or a regenerating organ. If you have a broken joint or bone then you have by definition mechanical signals; your muscles contract and there will be some sort of mechanical response. One knows that cells respond to that. But they also respond to various growth factors. What is not entirely clear is how these things work. For example, your mechanical signaling would tend to force a cell or an organ to go down process A, and chemical signaling forces it to go down process B. At what point do you switch from one to the other? These sorts of things are difficult to isolate. Indeed it is quite likely that, if you make the right combination, you might get a tissue to go down process $\mathrm{C}$, which goes in a completely different direction.

It is interesting that even with static materials - although you could also say that they are in some sense active - there is this point where you can do experiments on a material and calculate the stress to rupture. What is the loading the material can handle? You can design your object for this type of loading. You can then do the same sort of experiments for a corrosive environment for example. So we need to be able to measure the concentration of acid that a material can withstand before it begins to be eaten away. Following on this, you can - as an engineer - put in your design conditions based on these two criteria and build your components. In some fairly well-known examples, you get to the point where your materials start breaking at stresses and concentrations much lower than what you planned for because you have these combined phenomena [Hänninen 2003]. This process is called stress corrosion cracking, which has been found to be responsible for some pipeline and bridge failures. It means that you must develop whole new scenario criteria to describe this process.

This is what is being done in engineering and the same is required for understanding this in biology. It is known that mechanics can influence cell behavior. But it is also known that mechanics can influence chemistry. There is this great work from Viola Vogel's Laboratory of Applied Mechanobiology at the ETH Zürich where they look at the unfolding of protein complexes when you load them with force. ${ }^{9}$ You can see changes in the chemical activity of these complexes due to the presence

8 See [Grinthal and Aizenberg 2013, p. 7072]; see also the interview with Joanna Aizenberg in this volume.

9 For the research of the Laboratory of Applied Mechanobiology, see online: https://appliedmecha nobio.ethz.ch/ (accessed May 5, 2021). See also: [Vogel 2006; Vogel and Sheetz 2009]. 
of external forces. This gives rise to a molecular mechanism whereby mechanical signaling can change local chemistry and mechanics. Then you have this very interesting feedback effect (see Fig. 5) in which cell response to a physical environment changes that environment.

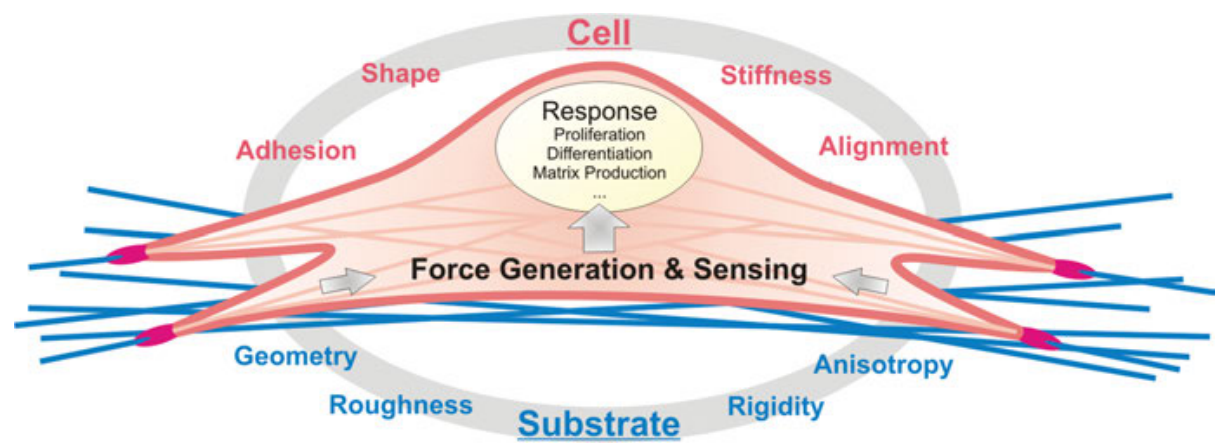

Fig. 5: Cells attached to a substrate respond to its physical properties via the generation of forces applied by the cytoskeleton to the focal adhesions. These mechanical signals give rise to responses in cell behavior, for example with changes in proliferation, differentiation, and extracellular matrix production. From: [Kollmansberger et al. 2011, fig. 1]. Reproduced by permission of The Royal Society of Chemistry.

Whether this feedback process is deterministic or not, I think one could say that the process by which you load up a molecule and describe force displacement or the unfolding process is one thing, and the process by which the chemical reactions would occur is another thing, but in the end you have to describe both together in one system to be able to be predictive.

I think that there is still a lot of interesting work to be carried out. This is also true in the applied direction, where people look at these so-called 'self-healing materials' - which in some sense you could also describe as being a component of active materials - that will respond to the presence of a defect by allowing new material to polymerize and reseal the crack. These filler materials can fill the gap either by surface tension, or by being actively pumped into the fracture gap of an engineering object [Hager, Zwaag, and Schubert 2016; Thomas and Surendran 2020]. But they have also very interesting feedback effects, because the healing material is never going to be the same as the original material that was there. Due to the fact that in your first cycle you can crack and heal, it is quite likely that in the second cycle the behavior will be different. Looking at this over a long period of time goes in the same direction. You need to consider both. Not only the chemistry of the healing process but also the mechanics of the cracking process and how all of these fit together. That would be a fascinating addition to this concept of active matter, which I had not really thought about before. In this respect, healing would be part of that as well. 
KK: When you are dealing with living systems it is not only the relation of chemistry and mechanics that is relevant but also the role of genetics. What happens to the feedback dance if you manipulate the genetics of cells? How does this affect the chemical signaling, geometric signaling, or mechanical signaling?

JD: The classical, neo-Darwinistic style of describing living systems would say that everything is written in the genetic code. Maybe that viewpoint of how shape appears in biology is not entirely fair with respect to neo-Darwinism, in the sense that there it is more a question that the genetic code gives rise to some sort of organism structure, which then is more or less successful, and if it survives it can pass these genes further to the next generation. The shape itself is in some way irrelevant unless it has got something to do with the fitness. You could imagine two genetically identical trees: one you grow down in the valley floor, one up in a crack in a cliff face. Due to differences in environment, they will create very different forms. Due to the constraints of the rock and high winds, one plant might be highly deformed, leaning to one side, while the tree in the plains or protected environment grows high and straight. What is clear is that the environmental constraints can clearly influence the shape. From that perspective, you could say that genetics gives an organism the tools whereby the various processes can occur in reaction to the various environmental constraints, which can be immense in size.

I think one key point is related here to what Thompson in his book On Growth and Form wrote on living organisms: they operate in a physical world so there are physical constraints and as such these constrains will put limitations on what is actually possible. ${ }^{10}$ That being said, the growing organism will be acting against that. Through the influx of energy into a system, through the influx of molecules from the surroundings, an organism will be able to create shapes. But, if you have a floppy membrane surrounding a single cell, the membrane has a certain bending energy, a certain stretching energy. This will have an influence on the sorts of shapes that it can then achieve. The same goes for a growing organism: there are certain processes that will occur, but these processes are constrained inside this physical world. The key point in general is to find out to what extent these constraints influence the sorts of processes that occur. If you go back to the swarming models of Tamás Vicsek and others [Vicsek 1995, footnote 7], if you allow swarming in confined spaces, the fact that you have physical walls or you can constrain objects to a curved surface will influence the sort of patterns in self-organization that you can achieve. This is again

10 See [Thompson 1945, p. 10]: "Cell and tissue, shell and bone, leaf and flower, are so many portions of matter, and it is in obedience to the laws of physics that their particles have been moved, moulded and conformed. [...] Their problems of form are in the first instance mathematical problems, their problems of growth are essentially physical problems, and the morphologist is, ipso facto, a student of physical science." 
something you will observe with cells, bacteria, or the patterning of living interacting objects in space.

I do not know if it is the environment influencing the genetics, although there are studies that show that in principle this is possible - in epigenetics say. The view of how organisms can change with time is undergoing expansion. But what we are in principle focusing on is how this given organism responds in terms of its shape to the physical environment. The driving force, if you like, for shape changes, which would consist of how cells interact with their environment. If you provide constraints, this can then influence on how the cells organize. If you give an anisotropic environment where cells are aligned on a fiber-like structure, they will also align, but, if they then start producing extra cells in a matrix, such materials will also have an influence [Bidan et al. 2016; Ehrig et al. 2019]. They will be influenced by the orientation of the cells, which will then feedback on the next layer of cells that will feel again this anisotropic environment. You have this intense feedback process occurring between environmental conditions and behavior of cells. Environmental conditions are the local conditions around cells and growing tissues, which will then change the local physical environment once again. This type of feedback process is very interesting. If you start pushing cells in a certain direction, you can try to get them aligned in a particular way. Your genetic description would say that they would try to switch back to their desired or idealized direction. To what extent would this mechanical or geometrical signal then override what is occurring at the genetic level? I am not sure that this has been really understood.

KK: I would like to come back to the relation of living matter to its environment in a different way. Living entities are described in energetic terms as open systems and, moreover, as systems far from equilibrium, because they are incessantly dependent on energy and material inflows from their environment and therefore have to come to terms with a wide variety of environments. Living systems adapt either by changing their internal structures or by building external structures to regulate the influx - just as the beaver constructs a burrow and thus adapts the environment for itself, creating its own partially stable niche. The biofilm of bacteria would be another example. J. Scott Turner has written on this kind of 'niche construction,' and he describes it as a "[p]hysiology of the [e]nvironment" [Turner 2000, p. 7]. ${ }^{11}$ What I am heading at is: because living systems are open systems, they have the necessity and the possibility to actively design their boundaries, but they cannot fully close these boundaries - and this means that they always have to deal with instabilities, both in a positive sense (they evolve by adapting to changing conditions) and in a negative sense (too much instability can destroy the system). I wonder how this productive role of instability in structural development and environmental relations is reflected in the research on active materials?

11 For an application of Turner's ideas on biofilm (as an architecture that balances the instabilities that appear because of changes in the environment), see: [Hengge 2020]. 
JD: There is wonderful work on instabilities done in and around the group of Lakshminarayanan Mahadevan from the Department of Physics at Harvard University. ${ }^{12}$ In his Soft Math Lab, they looked at patterning - say of the villi in the lining of the gut or stomach (see Fig. 6). What they could show was that the sort of complex oscillations could be genetically determined, but the immediate physical cause was a buckling instability [Savin et al. 2011]. ${ }^{13}$ Picking up your word instability here, this is a mechanical instability, which is then coupled back to growth processes and other similar things.

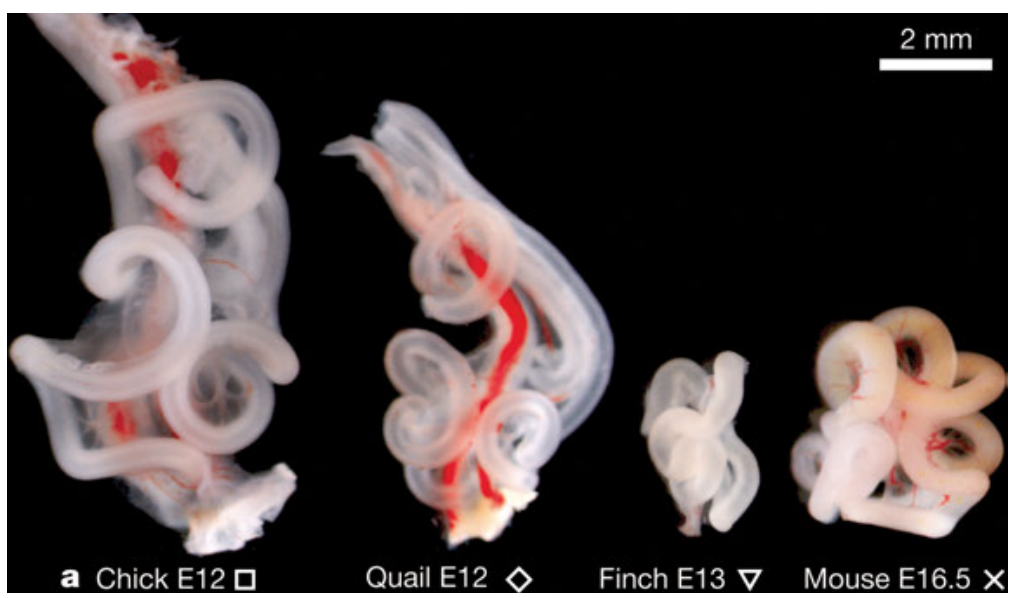

Fig. 6: Gut looping patterns in the chick, quail, finch, and mouse (to scale). The comparison shows qualitative similarities in the shape of the loops. Reprinted by permission from Springer Nature: [Savin et al. 2011, fig. 5a].

From the perspective of physics or mathematics, what is nice about these kinds of concepts is that you do not need that much information to create a very complex structure. You have this very complex patterning that you see, highly convoluted surfaces, but in principle, it comes from relatively simple phenomena. The information that an organism needs is what thickness of tissue needs to be grown on what other thickness of other tissue - and then due to differential growth you come up with this pattern formation. And it comes automatically out. That is a very efficient way of coding for structure. You do it in the properties of the materials that are stuck together and how they are arranged in space and that gives you an extra dimension to work with. This feedback effect between your external structure and your structure

12 https://softmath.seas.harvard.edu/ (accessed May 5, 2021).

13 For an application of this research to the brain, see also: [Tallinen et al. 2016]. 
which is influenced by your organism. The examples of Scott Turner are something that is fascinating to explore, because the example of structures such as nests and burrows built or in some way "grown" by animals can then give rise to very interesting pattern formation at much larger length scales than what one would expect if it was just deterministic growth alone. It becomes much more efficient in terms of the information storage that is required, because it is built into the physics which are already there in the environment or in our world that we are living in.

KK: My last question aims at the future horizon of the research on active materials. What are the next challenges in this field of research?

JD: A big challenge that I would at least like to be involved with is the question of how extracellular matrix components organize overlarge length scales, giving rise to certain types of structures, function, and form. Again, this is about internal structures of tissues. What is fairly well understood is how a certain arrangement of component materials gives rise to a certain function. That you can do because it is a static situation. But then how does this actually come to be? That is a big question. Liquid crystal modeling here is very interesting because it seems to suggest at least that there is a certain self-organization process that is occurring in controlling that. An alternative is to use agent-based models that can explicitly deal with the extracellular matrix that is produced when tissue grows. This would be for example collagen or fibronectin when you are talking about bone or skin. In plants, you have cellulose microfibrils, chitin in arthropods, all of which are further complicated by the mineralization process that may occur in the environment created by cells. How these extracellular matrix components are organized in space will have an influence on the properties of the environment for future generations of cells. So again, you have this feedback effect. Describing this at local level is incredibly difficult. Finding a way of accomplishing that is certainly part of the research of the future. It is a question of coming up with suitable experiments where you can really test these ideas in 3D. I do not see much that is being done or fully understood in this direction. One other way of describing the patterns that are observed in these extracellular matrix components is to make analogies with other sorts of similar patterns that are being observed in synthetic material, for instance in liquid crystals [Gompper et al. 2020]. There is the work of Pierre-Gilles de Gennes and Jacques Prost, who have pushed forward this idea, but observing these processes live would be a key step in understanding [De Gennes and Prost 1993]. Why is that important? It is important in terms of basic biology and also in terms of medicine and in terms of the understanding of certain types of diseases or regeneration processes. Ideally, you want tissues to regenerate in the patterning they had beforehand. If they do not you sort of end up with a scar tissue with different properties, and that can be problematic. If one has an understanding of how these sorts of organizational processes during growth or during remodeling of tissues 
actually occur, then you can maybe apply that to artificial systems of course. ${ }^{14}$ That could be an ultimate dream.

What one can always win from studying active matter - and in particular if you go in the direction of understanding how the physical constraints surrounding this complex interacting system can have a huge effect on how growth occurs - is how patterning occurs at different length scales and different levels. Studies in this direction can have very wide implications. If, for instance, you look at the geometric constraints here in Salzburg, where you have mountains that surround the area, and regions where you cannot build, that means the town is built around certain types of constraint, which have an effect on things like transport and how the town can grow. It is not just a deterministic process where the growth of a town is in the hands of city planners. The surroundings have an implicit effect on how growth can occur. And this is something that is generalizable over many length scales and many different fields. Probably in the social sciences you could also have these sorts of concepts where constraints due to certain topics that are not able to be discussed in a particular environment might force certain ideas to come out preferentially. For example, constraints of media: the constraint of the way we read, the constraint of a printed book versus a digital object - these constraints change how that media can actually be presented - the constraints of film, where in principle you will not rewind to see things again, because typically it goes from start to finish without any or much interaction. These sorts of things are all linked to the studying of active matter.

\section{Bibliography}

Ambrosi D, Ateshian GA, Arruda EM et al. Perspectives on Biological Growth and Remodeling. Journal of the Mechanics and Physics of Solids 2011, 59 (4), 863-883. DOI: 10.1016/j. jmps.2010.12.011.

Bidan CM, Kollmannsberger P, Gering V et al. Gradual Conversion of Cellular Stress Patterns into Pre-Stressed Matrix Architecture During in Vitro Tissue Growth. Journal of the Royal Society Interface 2016, 13 (118), 20160136, DOI: 10.1098/rsif.2016.0136.

Bidan CM, Kommareddy KP, Rumpler M et al. How Linear Tension Converts to Curvature: Geometric Control of Bone Tissue Growth. Plos One 2012, 7 (5), e36336. DOI: 10.1371/journal.pone.0036336.

Burgert I. Plant Biomechanics and Biomimetics. Munich: Max Planck Institute of Colloids and Interfaces, (2021. Accessed May 5, 2021, at https://www.mpikg.mpg.de/5788791/plantbiomechanics-and-biomimetics.)

Burgert I, Dunlop JWC. Micromechanics of Cell Walls. In: Wojtaszek P, ed. Mechanical Integration of Plant Cells and Plants. Series: Signaling and Communication in Plants. Vol. 9. Berlin, Heidelberg, Springer, 2011, 27-52, DOI: 10.1007/978-3-642-19091-9_2.

14 Ideas of this sort can be seen in the work of André Studart (ETH Zürich), who tries to mimic biological structures to create new functional materials [Ferrand et al. 2015]. 
Camazine S, Deneubourg JL, Franks NR, Sneyd J, Theraula G, Bonabeau G. Self-Organization in Biological Systems. Princeton, Princeton University Press, 2003.

Dunlop JWC. The Physics of Shape Changes in Biology. Habilitationsschrift zur Erlangung der Lehrbefähigung für das Fach Physik. University of Potsdam, October 2015. (Accessed May 5, 2021, at: https://publishup.uni-potsdam.de/opus4-ubp/frontdoor/deliver/index/docld/9655/ file/dunlop_habil.pdf.)

Dunlop JWC. Biomimetic Actuation and Tissue Growth. Munich: Max Planck Institute of Colloids and Interfaces, (2021. Accessed May 3, 2021, at https://www.mpikg.mpg.de/5896344/Biomi metic-Actuation-and-Tissue-Growth.)

Dunlop JWC, Bréchet Y, Legras L. A Coupled Recovery/Recrystallisation Model for Zirconium Alloys. Influence of Hydrogen. Materials Science Forum 2004, 467-470, 629-634. DOI: 10.4028/www. scientific.net/MSF.467-470.629.

Dunlop JWC, Fischer FD, Gamsjäger E, Fratzl P. A Theoretical Model for Tissue Growth in Confined Geometries. Journal of Mechanics and Physics of Solids 2010, 58 (8), 1073-1087. DOI: 10.1016/j.jmps.2010.04.008.

Dunlop JWC, Fratzl P. Multilevel Architectures in Natural Materials. Scripta Materialia 2013, 68 (1), 8-12, DOI: 10.1016/j.scriptamat.2012.05.045.

Dunlop JWC, Ionov L. Shape-Programmed Folding of Stimuli-Responsive Polymer Bilayers. ACS Nano 2012, 6 (5), 3925-3934, DOI: https:/doi.org/10.1021/nn300079f.

Dunlop JWC, Weinkamer R, Fratzl P. Artful Interfaces within Biological Materials. Materials Today 2011, 14 (3), 70-78, DOI:10.1016/S1369-7021(11)70056-6.

Dunlop JWC, Zickler GA, Weinkamer R, Fischer FD, Fratzl P. The Emergence of Complexity from a Simple Model for Tissue Growth. Journal of Statistical Physics 2020, 180, 459-473, DOI: 10.1007/s10955-019-02461-7.

Ehrig S, Schamberger B, Bidan CM et al. Surface Tension Determines Tissue Shape and Growth Kinetics. Science Advances 2019, 5 (9), eaav9394, DOI: 10.1126/sciadv.aav9394.

Ferrand HL, Bouville F, Niebel TP, Studart AR. Magnetically Assisted Slip Casting of Bioinspired Heterogeneous Composites. Nature Materials 2015, 14 (11), 1172-1179.

Fischer FD, Zickler GA, Dunlop JWC, Fratzl P. Tissue Growth Controlled by Geometric Boundary Conditions: A Simple Model Recapitulating Aspects of Callus Formation and Bone Healing. Journal of the Royal Society Interface 2015, 12 (107), 20150108, DOI: 10.1098/rsif.2015.0108.

Fratzl P, Barth FG. Biomaterial Systems for Mechanosensing and Actuation. Nature 2009, 462, 442-448, DOI: 10.1038/nature08603.

Fratzl P, Weinkamer R. Nature's Hierarchical Materials. Progress in Materials Science 2007, 52 (8), 1263-1334, DOI: 10.1016/j.pmatsci.2007.06.001.

Gamsjäger E, Bidan CM, Fischer FD, FratzI P, Dunlop JWC. Modelling the Role of Surface Stress on the Kinetics of Tissue Growth in Confined Geometries. Acta Biomaterialia 2013, 9 (3), 5531-5543, DOI: 10.1016/j.actio.2012.10.020.

Gennes P-G de, Prost J. The Physics of Liquid Crystals, Oxford, Clarendon Press, 1993.

Gompper G, Winkler RG, Speck T et al. The 2020 Motile Active Matter Roadmap. Journal of Physics: Condensed Matter 2020, 32, 193001, DOI: 10.1088/1361-648X/ab6348.

Grégoire G, Chaté H. Onset of Collective and Cohesive Motion. Physical Review Letters 2004, 92 (2), 025702, DOI: 10.1103/PhysRevLett.92.025702.

Grinthal A, Aizenberg J. Adaptive all the Way Down: Building Responsive Materials from Hierarchies of Chemomechanical Feedback. Chemical Society Reviews 2013, 42 (17), 7072-7085, DOI: 10.1039/C3CS60045A.

Guiducci L, Weaver JC, Bréchet YJM, Fratzl P, Dunlop JWC. The Geometric Design and Fabrication of Actuating Cellular Structures. Advanced Materials Interfaces 2015, 2 (11), 1500011, DOI: 10.1002/admi.201500011. 
Hager MD, Zwaag S van der, Schubert US. Self-Healing Materials. Cham, Springer, 2016.

Halatek J, Frey E. Rethinking Pattern Formation in Reaction-Diffusion Systems. Nature Physics 2018, 14, 507-514, DOI: 10.1038/s41567-017-0040-5.

Hänninen HE. Stress Corrosion Cracking. In: Milne I, Karihaloo B, Ritchie RO, eds. Comprehensive Structural Integrity. Vol. 6. Environmentally Assisted Fatigue. Ed. Petit J, Scott PM. Amsterdam et al., Elsevier, 2003, 1-29.

Harrington MJ, Razghandi K, Ditsch F et al. Origami-Like Unfolding of Hydro-Actuated Ice Plant Seed Capsules. Nature Communications 2011, 2, 337, DOI: 10.1038/ncomms1336.

Hengge R. Linking Bacterial Growth, Survival, and Multicellularity - Small Signaling Molecules as Triggers and Drivers. Current Opinion in Microbiology 2020, 55, 57-66, DOI: 10.1016/j. mib.2020.02.007.

Huss JC, Fratzl P, Dunlop JWC, Merritt DJ, Miller BP, Eder M. Protecting Offspring Against Fire: Lessons from Banksia Seed Pods. Frontiers in Plant Science 2019, 10, 283, DOI: https:/doi. org/10.3389/fpls.2019.00283.

Khan A, Jawaid M, Raveendran SN, Asiri AMA, eds. Self-Healing Composite Materials. From Design to Applications. Duxford et al., Woodhead Publishing, Elsevier, 2019/2020, DOI: 10.1016/ C2018-0-02118-0.

Kollmannsberger P, Bidan CM, Dunlop JWC, Fratzl P. The Physics of Tissue and Extracellular Matrix Organisation: How Cells Join Forces. Soft Matter 2011, 7 (20), 9549-9560, DOI: 10.1039/ C1SM05588G.

Kondo S. The Reaction-Diffusion System: A Mechanism for Autonomous Pattern Formation in the Animal Skin. Genes to Cells 2002, 7 (6), 535-541. DOI: 10.1046/j.1365-2443.2002.00543.x.

Lecuit T, Lenne PF. Cell Surface Mechanics and the Control of Cell Shape, Tissue Patterns and Morphogenesis. Nature Reviews Molecular Cell Biology 2007, 8, 633-644. DOI: 10.1038/ nrm2222.

Malacincski GM, Bryant SV, eds. Pattern Formation. A Primer in Developmental Biology. New York, London, Macmillan Publishing Company, Collier Macmillan Publishers, 1984.

Meinhardt H, Klingler M. A Model for Pattern Formation on the Shells of Molluscs. Journal of theoretical Biology 1987, 126 (1), 63-89, DOI:10.1016/S0022-5193(87)80101-7.

Menon GI. Active Matter. In: Krishnan JM, Deshpande AP, Kumar PB, eds. Rheology of Complex Fluids. New York, Springer, 2010, 193-218.

Nüsslein-Volhard C, Singh AP. How Fish Color their Skin: A Paradigm for Development and Evolution of Adult Patterns. BioEssays 2017, 39 (3), 1600231, DOI: 10.1002/bies.201600231.

Reyssat E, Mahadevan L. Hygromorphs: From Pine Cones to Biomimetic Bilayers. Journal of The Royal Society Interface 2009, 6 (39), 951-957, DOI: 10.1098/rsif.2009.0184.

Rumpler M, Woesz A, Dunlop JWC, Dongen JT van, Fratzl P. The Effect of Geometry on ThreeDimensional Tissue Growth. Journal of the Royal Society Interface 2008, 5 (27), 1173-1180, DOI: $10.1098 /$ rsif.2008.0064.

Savin T, Kurpios NA, Shyer AE, Florescu P, Liang H, Mahadevan L, Tabin CJ. On the Growth and Form of the Gut. Nature 2011, 476, 57-62, DOI: 10.1038/nature10277.

Stoychev G, Zakharchenko S, Turcaud S, Dunlop JWC, Ionov L. Shape-Programmed Folding of StimuliResponsive Polymer Bilayers. ACS Nano 2012, 6 (5), 3925-3934, DOI: 10.1021/nn300079f.

Stoychev G, Turcaud S, Dunlop JWC, Ionov L. Hierarchical Multi-Step Folding of Polymer Bilayers. Advanced Functional Materials 2013, 23 (18), 2295-2300, DOI: 10.1002/adfm.201203245.

Tallinen T, Chung JY, Rousseau F, Girard N, Lefèvre J, Mahadevan L. On the Growth and Form of Cortical Convolutions. Nature Physics 2016, 12, 588-593. DOI: 10.1038/nphys3632.

Thomas S, Surendran A, eds. Self-Healing Polymer-Based Systems. Amsterdam, Oxford, Cambridge MA, Elsevier, 2020, DOI: 10.1016/C2018-0-03002-9. 
Thompson DW. On Growth and Form. Cambridge, New York, Cambridge University Press, The Macmillan Company, 1945.

Trepat X, Sahai E. Mesoscale Physical Principles of Collective Cell Organization. Nature Physics 2018, 14 (7), 671-682, DOI: 10.1038/s41567-018-0194-9.

Turcaud S, Guiducci L, Fratzl P, Bréchet YJM, Dunlop JWC. An Excursion into the Design Space of Biomimetic Architectured Biphasic Actuators. International Journal of Materials Research 2011, 102 (6), 607-612, DOI: 10.3139/146.110517.

Turing AM. The Chemical Basis of Morphogenesis. Philosophical Transactions of the Royal Society B 1952, 237 (641), 37-72, DOI: 10.1098/rstb.1952.0012.

Turner JS. The Extended Organism: The Physiology of Animal-Built Structures. Cambridge MA, London, Harvard University Press, 2000.

Vicsek T, Czirók A, Ben-Jacob E, Cohen I, Shochet O. Novel Type of Phase Transition in a System of Self-Driven Particles. Physical Review Letters 1995, 75 (6), 1226-1229, DOI: 10.1103/ PhysRevLett.75.1226.

Vogel V. Mechanotransduction Involving Multimodular Proteins: Converting Force into Biochemical Signals. Annual Review of Biophysics and Biomolecular Structure 2006, 35 (1), 459-488, DOI: 10.1146/annurev.biophys.35.040405.102013.

Vogel V, Sheetz MP. Cell Fate Regulation by Coupling Mechanical Cycles to Biochemical Signaling Pathways. Current Opinion in Cell Biology 2009, 21 (1), 38-46, DOI: 10.1016/j. ceb.2009.01.002.

Zienkiewicz OC, Taylor RL, Zhu JZ. The Finite Element Method: Its Basis and Fundamentals. 6th ed. Amsterdam et al., Elsevier Butterworth-Heinemann, 2005.

Zurob HS, Bréchet Y, Dunlop J. Quantitative Criterion for Recrystallization Nucleation in Singlephase Alloys: Prediction of Critical Strains and Incubation Times. Acta Materialia 2006, 54 (15), 3983-3990. DOI: 10.1016/j,actamat.2006.04.028.

John Dunlop studied chemistry and materials engineering at the University of Western Australia, before he moved to Grenoble, France, to start a PhD in Metal Physics with Yves Bréchet. There he studied the microstructure of and the physical changes that could occur in spent nuclear fuel rods during transport. After his PhD, he moved to the MPICl in Potsdam, Germany, to the Department of Biomaterials of Peter Fratzl. After the end of his Humboldt fellowship, he stayed on in Potsdam for almost 10 years working as a group leader researching issues related to understanding the physics of shape changes in living and dead materials. Since 2017, John Dunlop is professor for 'Biological Physics' at the University of Salzburg and heads the 'MorphoPhysics' group there, continuing to work on a variety of topics related to active materials. 
